ship and the "specialness" of the attention that they received. Many patients cope by avoiding or denying their sexual feelings. ${ }^{9}$

Others, feeling uncomfortable and unsafe with their sexuality, use it in chaotic, inappropriate, and self defeating ways. In working through feelings of shock, revulsion, and anger and re-examining issues of power, violence, and sexism within society doctors may find their own sexual relationships affected and may go through a stage where they see abuse everywhere. ${ }^{10}$ They may find themselves "turned on" as much as "turned off" by accounts of the abuse and mistrust their own motives.

The power and intensity of emotion experienced by both patient and doctor in the therapeutic relationship can be disturbing. Some of these feelings may remain unconscious but get acted on to the detriment of treatment-for example, doctors may inappropriately discharge or over investigate the patient. If the doctor can try to understand what is going on in his or her relationship with the patient and recognise the feelings that emerge as important clues about the patient's childhood then managing the patient will be far more rewarding and the patient's prognosis will be substantially improved.

I thank Dr Chris Whyte for his many helpful comments.

Senior Registrar in Psychotherapy,

PENELOPE CAMPLING

Humberstone Grange Clinic,

Leicester LE5 0TA

1 Sheldon $\mathrm{H}$. Child sexual abuse in adult female psychotherapy referrals: incidence and implicatio for treatment. Br f Psychiatry 1988;152:107-11.

Hall Z. Understanding women in distress. London: Tavistock/Routledge, 1989

3 Gardener F. Psychotherapy with adult survivors of child sexual abuse. British fournal Psychotherapy 1990;6:285-94

4 MacCarthy B. Are incest victims hated? Psychoanalytic Psychotherapy 1988;3:113-20.

5 Fountain DM, Moore B. Therapy for adult victims of childhood sexual abuse in a district setting Psychiatric Bulletin 1989;13:437-9.

6 Davenport S, Sheldon H. From victim to survivor. Changes 1987;5:379-82.

7 Winnicott DW. Hate in the countertransference. Int $\mathcal{F}$ Psychoanal 1949;30:60-7

Cahill C, Llewelyn SP, Pearson C. Treatment of sexual abuse which occurs in childhood: A reviewo Brf Clin Psychol 1991;30:1-12.

9 John D. Beyond sexual abuse: therapy with women who were victims in childhood. Chichester: Wiley, 1980

10 Perry J. Sexual abuse-the counsellor's process. Changes. 1989;7(3):93-4.

\title{
How general practitioners can help subfertile couples
}

\section{Support, early investigation, and referral}

Subfertility affects one couple in $10^{1}$ and may cause as deep and enduring a sense of loss as many chronic illnesses. ${ }^{2}$ Commissioning authorities and general practitioners should therefore take subfertility seriously. Fortunately, the condition is starting to receive the attention that it deserves, and two important documents have recently been published on its management. ${ }^{34} \mathrm{~A}$ database of clinical trials incorporating structured overviews and meta-analyses has been established jointly in Leeds and Hamilton, Ontario, and is funded by the Yorkshire research and development programme and the Canadian Royal Commission on Reproductive Technologies.

Where do general practitioners fit into the diagnosis and management of infertility? Firstly, general practitioners can provide the emotional support that most infertile couples need before, during, and after treatment, whether successful or not. Subfertility evokes powerful and complex emotions, which can make it difficult for couples to assimilate spoken information. Yet such information is crucial if they are to make fully informed decisions about their treatment. Although hospital clinics may provide written information and dedicated counsellors to help patients over particularly difficult times (for example, when assisted conception has failed), this cannot replace the general practitioner, with whom the couple are likely to have a longstanding relationship.

It is the general practitioner who will guide the couple to secondary care, having already listened to their history, examined them, and undertaken initial investigations. He or she can smooth the progress of investigation and act as a valuable source of advice and explanation during what may be complex and intensive treatments, ensuring that crucial information, such as the risks and consequences of twins and higher order births, have been fully assimilated.

Finally, it may be the general practitioner who has to help the couple face and come to accept their permanent childlessness. Such involvement was much hindered by the requirements of confidentiality in the Human Embryology and Fertilization Act, which, before the recent amendment, kept general practitioners unaware of their patients' treatment. General practitioners can help by reducing the number of journeys to distant clinics, as well as acting as the patient' advocate and providing continuity.

The main issues for general practitioners concern how much investigation they should carry out, when and to whomes they should refer the patient (local hospital or tertiary centre)? and how much they should be involved in on going treatmento (for example, giving drugs to stimulate ovulation). Far toos many patients are brought back time and again to outpatiento clinics where they are subjected to an unending series of investigations, ${ }^{5}$ most of which have little hope of improving their chance of success. General practitioners, in their role aso purchaser and patient's advocate, are responsible for guard-ing against this. Genuine remediable causes are found in lesso. than half the cases of subfertility. ${ }^{6}$ The general practitioner's role is therefore twofold: firstly, to enable the couple to find out whether a specific cause exists and to refer accordinglyo and, secondly, to give patients the information that they need to decide when, if ever, they wish to pursue assistedo conception procedures.

Known causes of subfertility can be divided into failure to요 ovulate, defective sperm, and tubal damage. General practitioners can exclude failure to ovulate by taking a carefully timed blood sample for measurement of the progesterone concentration. This must be interpreted with reference to the date of the succeeding menstrual period. Semen analyses can $N_{\omega}$ exclude most abnormalities of the sperm; if the sperm count iso initially low it should be repeated. Ideally, the sperm shouldo be analysed by the laboratory connected to the centre to which the patients will be referred if further investigation or treatment is necessary. Tubal damage can be assessed laparoscopically, and a set protocol and method of documenting the findings should be used. Couples prefer to be seen in designated subfertility clinics rather than general outpatientoclinics.

Some regions have drawn up protocols for investigation and treatment by general practitioner, nonspecialist, and specialist hospitals. A randomised study in Grampian has shown that published guidelines are effective in improving referral practice and cutting down unnecessary investigations (C Emslie et al, unpublished data). 
If no obvious cause for subfertility is found then the timing of referral to a specialist centre is important and should take account of the woman's age and the duration of subfertility. The longer the patient remains childless the lower the chance of conception, and as time runs out anxiety increases. Purchasing authorities are beginning to recognise the importance of subfertility and to purchase services for assisted conception. The techniques themselves are coming under increasing scrutiny from randomised trials. For example, 10 such studies have evaluated the effects of down regulation of the pituitary gland before ovulation was induced, and these have confirmed that this treatment is effective in doubling the rate of conception and halving the number of cancelled cycles.

General practitioners need information about the effectiveness of treatments for subfertility so that they can advise patients and contribute to any discussion about the level of funding of subfertility services. The newly published bulletin on the management of subfertility ${ }^{3}$ and the guidelines from the Royal College of Obstetricians and Gynaecologists on the topic ${ }^{4}$ will help such difficult choices.
We thank Professor Allan Templeton of Aberdeen and Dr Patrick Vandekerckhove of Leeds for their helpful comments.

RICHARD LILFORD

Professor of Obstetrics and Gynaecology

Department of Clinical Medicine,

University of Leeds,

Leeds LS2 9LN

GAVIN YOUNG General Practitioner

The Surgery,

Barn Croft,

Temple Sowerby,

Penrith CA10 1RZ

1 Greenhall E, Vessey M. The prevalence of subfertility: a review of the current confusion and a report of two new studies. Fertil Steril 1990;54:978-83.

2 Edelmann RJ, Connolly KJ. Psychological aspects of infertility. Br F Med Psychol 1986;59:1-1

3 The management of subfertility. Effective Health Care. 1992; No 3(August): 1-24

Fertility Committee of the Royal College of Obstetricians and Gynaecologists. Infertility: guideline for practice. London:RCOG Press, 1992.

5 Bromham D, Balmer B, Clay R, Hamer R. Disenchantment with infertility services: a survey of patients in Yorkshire. Br f Family Planning 1988;14:3-8.

6 Lilford RJ, Dalton ME. Effectiveness of treatment for infertility. BMF 1987;295:155-6.

7 Hughes E, Fedorkow D, Daya S, Sagle M, Vandekerckhove P, Collins J. The routine use of gonadotropin releasing hormone agonists prior to IVF and GIFT: a meta-analysis of randomized controlled trials. Fertil Steril (in press).

\title{
Part time working and job sharing in the NHS
}

\author{
Some worthwhile progress but still a long way to go
}

More than a million people work in the NHS and three quarters of them are women. Faced with such a large female workforce and pressure from the Equal Opportunities Commission, ${ }^{1}$ the NHS Management Executive has created the NHS Women's Unit and issued a handbook of good practice, ${ }^{2}$ which includes large sections on improving opportunities for part time working. Because of their domestic commitments many women need to work part time and will sacrifice their career prospects, turning to unpopular specialties and non-career grades to balance their family and career. $^{3}$

Part time working for doctors in the hospital service has had to fit into tight controls on staffing and has proceeded from the top down. Since the 1960s there have been several central directives, leading to the current nationally organised training schemes for registrars and senior registrars. ${ }^{45}$ Under the senior registrar scheme (PM(79)3) candidates are interviewed annually for a limited number of places in each specialty. Those successful in gaining manpower approval must arrange training with a local unit, convince the relevant college of their appointment's educational value, and get funds from the region. Just over a fifth of female senior registrars have graduated from this crash course in NHS bureaucracy and are working part time (Department of Health, personal communication). Of course, half time often means a 40 hour week. The faults of PM(79) 3 have been appreciated, and the new registrar arrangements $(\mathrm{EL}(91) 5)$, although similar, have been streamlined and are centrally funded, at least until 1995. Currently $11 \%$ of female registrars work part time, and the aim is to quadruple that number in five years. For senior house officers health authorities and trusts can set up part time posts without applying for central approval; $5 \%$ of female senior house officers work part time. No formal part time schemes exist for consultants, and full time working is the norm, with only $9 \%$ of all consultants having an NHS commitment of eight sessions or less.

In general practice trainees can work part time for two years, and about one in 10 women do so, with trainers getting half the training allowance. For principals in general practice partners may be full time, three quarter time, or half time. Around $15 \%$ of female principals are three quarter time partners, and fewer than $3 \%$ are half time. ${ }^{6}$ The retainer scheme is the medical profession's only scheme for managing a career break and is used by just 550 women, almost exclusively in general practice, doing up to two paid sessions a week.

There are no job sharing schemes as such, but the practice is now officially encouraged by the NHS Management Executive and has been a small scale success in different professions, specialties, and grades across the health service. ${ }^{8}$ It is attractive to employers, who do not have to restructure the service to accommodate part timers and who benefit from the bit more than half time that jobsharers do. Fears of excessive administrative and training costs and lack of continuity are largely unfounded. The big limitation is finding a partner and even with the job share registers set up by the BMA and several specialties this is likely to remain a minority solution for doctors. Some $1 \%$ of all principals in general practice now job share.

Comparing doctors with other NHS professionals-for example, the 320000 nurses-is interesting. In nursing, part time arrangements are evolving from the bottom up. Managers are offering staff a wide range of options-including limited hours, term time working, and local banks-and a third of nurses now work part time. This is usually, however, at the grade of staff nurse or below. Ward sisters wishing to work part time after maternity leave are frequently offered a lower grade, although sometimes job sharing will be explored at their former grade. ${ }^{9}$

Taken together, these options do not yet provide enough opportunities for part time working. Nearly three quarters of young women doctors would like to work part time when their children are young, ${ }^{10}$ but only $22 \%$ of female senior registrars are doing so. We must not forget the growing numbers of men 